\title{
$\mathrm{NiO} / \mathrm{PB}$ 复合电致变色薄膜的制备及其性能研究
}

\author{
岳言芳 ${ }^{1}$, 李海增 ${ }^{1}$, 李克点 ${ }^{1}$, 王金敏 ${ }^{2}$, 张青红 $^{3}$, 李耀刚 ${ }^{3}$, 陈 培 ${ }^{3}$, 王宏志 ${ }^{1}$
}

(1. 东华大学 材料科学与工程学院, 纤维材料改性国家重点实验室, 上海 201620; 2. 上海第二工业大学 环境与 材料工程学院, 上海 201209; 3. 东华大学 材料科学与工程学院, 先进玻璃制造教育部工程中心, 上海 201620)

摘 要: 采用水热法制备了垂直生长的氧化镍 $(\mathrm{NiO})$ 纳米片薄膜, 并利用电沉积法将普鲁士蓝(PB)负载到 $\mathrm{NiO}$ 纳米 片薄膜上，制备了新型的 $\mathrm{NiO} / \mathrm{PB}$ 复合电致变色薄膜。利用 $\mathrm{X}$ 射线衍射仪 $(\mathrm{XRD})$ 、场发射扫描电镜 $(\mathrm{SEM})$ 对样品的 晶型以及微观形貌进行了表征, 采用紫外-可见光光度计以及电化学工作站对 $\mathrm{NiO} / \mathrm{PB}$ 复合薄膜的电化学和电致 变色性能进行了研究和表征。结果表明: $\mathrm{NiO} / \mathrm{PB}$ 复合电致变色薄膜具有多孔结构和较大的比表面积, 可以增大 电解质与电极材料的接触面积。 $\mathrm{PB}$ 成功负载到 $\mathrm{NiO}$ 薄膜表面, 使 $\mathrm{NiO} / \mathrm{PB}$ 复合薄膜表现出较大的电流密度。相比于单 层 $\mathrm{NiO}$ 薄膜, $\mathrm{NiO} / \mathrm{PB}$ 复合薄膜表现出更好的电致变色性能, 其光调制范围可以达到 $46 \%$, 着色效率为 $141 \mathrm{~cm}^{2} / \mathrm{C}$, 并且其 着色时间可以缩短到 $5 \mathrm{~s}$, 裉色时间为 $6 \mathrm{~s}$ 。

关 键 词: $\mathrm{NiO} ; \mathrm{PB}$; 电致变色; 智能窗

中图分类号: TQ174 文献标识码: A

\section{Preparation and Properties of NiO/PB Hybrid Electrochromic Film}

\author{
YUE Yan-Fang ${ }^{1}$, LI Hai-Zeng ${ }^{1}$, LI Ke-Rui ${ }^{1}$, WANG Jin-Min ${ }^{2}$, ZHANG Qing-Hong ${ }^{3}$, \\ LI Yao-Gang ${ }^{3}$, CHEN Pei ${ }^{3}$, WANG Hong-Zhi ${ }^{1}$
}

(1. State Key Laboratory for Modification of Chemical Fibers and Polymer Materials, College of Materials Science and Engineering, Donghua University, Shanghai 201620, China; 2. School of Urban Development and Environmental Engineering, Shanghai Second Polytechnic University, Shanghai 201209, China; 3. Engineering Research Center of Advanced Glasses Manufacturing Technology, College of Materials Science and Engineering, Donghua University, Shanghai 201620, China)

\begin{abstract}
The vertically grown $\mathrm{NiO}$ hierarchical nano-structures were synthesized on FTO-coated glass using a crystal-seed-assisted hydrothermal technique. The NiO/PB film was obtained by the electrodeposition of $\mathrm{PB}$ on the $\mathrm{NiO}$ film. The crystal structures of the synthesis products were identified by X-ray diffraction technique and the microstructure and morphology of the films were characterized by field emission scanning electron microscope. The electrochemical property experiments were performed with an electrochemical workstation and the optical properties of the devices were recorded by Lambda 950. The NiO/PB film is composed of nanosheets and thus forming a porous structure. The porous structure could accelerate the interface electron kinetics between the film and electrolyte because its large electrochemical active area. Higher charge-insertion density of the NiO/PB film over the same period indicates faster kinetics, which means that the $\mathrm{NiO} / \mathrm{PB}$ film possesses fast electrochemical response. Compared with the single $\mathrm{NiO}$ film, the $\mathrm{NiO} / \mathrm{PB}$ film exhibits highly enhanced electrochromic performances, i.e. high optical modulation ( $46 \%$ at $700 \mathrm{~nm}$ without background correction), high coloration efficiency of $141 \mathrm{~cm}^{2} / \mathrm{C}$ at voltage of $2.5 \mathrm{~V}$, and fast
\end{abstract}

收稿日期: 2016-12-16; 收到修改稿日期：2017-02-08

基金项目: 国家自然科学基金(51672043，61674028,61376009); 广东省科技计划项目(2016B090932003) National Natural Science Foundation of China (51672043, 61674028, 61376009); Guangdong Province Science and Technology Program (2016B090932003)

作者简介：岳言芳(1992-), 女, 硕士研究生. E-mail: yanfangyue_2010@163.com

通讯作者: 王宏志, 教授. E-mail: wanghz@dhu.edu.cn 
response time of $6 \mathrm{~s}$ and $5 \mathrm{~s}$ for bleaching and coloration states, respectively.

Key words: NiO; PB; electrochromic; smart windows

$\mathrm{NiO}$ 作为电致变色器件的离子储存材料, 以其 较高的着色效率、较好的稳定循环性能以及较低的 材料合成成本等优点 ${ }^{[1]}$, 都得到广泛关注。但 $\mathrm{NiO}$ 的光调制范围相对较小, 只能在灰色和无色之间转 换, 限制了其应用。NiO 可以通过多种方法合成, 主 要包括磁控溅射法 ${ }^{[2]}$ 、电化学沉积法 ${ }^{[3]}$ 以及水热法 ${ }^{[4]}$ 等。 $\mathrm{NiO}$ 的电致变色过程依赖于离子的嵌入迁出过 程, 而多孔的结构形貌可以提供更大的反应活性面 积, 因此水热法一直都是研究热点 ${ }^{[5]}$ 。普鲁士蓝(PB) 是由 $\mathrm{Fe}^{2+}$ 与 $\mathrm{Fe}^{3+}$ 作为顶点排列成的立方晶格结构, $\mathrm{CN}^{-}$以直线排布作为立方体的棱边, 搭建起来的三 维网状结构可用作离子和电子的传输介质和通道。

PB 因其具有较大的光调制范围、较快的变色响应速 度以及较低的电压需求等优势, 成为近些年来研究 较为广泛的阳极电致变色材料 ${ }^{[6-7]}$ 。 PB 的制备方法 主要有电化学沉积法 ${ }^{[8]}$ 和溶液法 ${ }^{[9]}$ 。相比于溶液法, 电化学沉积法易得到分布均匀的 PB 薄膜, 薄膜与 基底的结合力较强, 因此研究者们常用电沉积法制 备 PB 电致变色薄膜 ${ }^{[10]}$ 。

单一电致变色材料的性能已经不能满足实际应 用的需求, 复合纳米电致变色材料已成为越来越受 关注的课题。复合的核心就是通过关联物质间结构、 形貌和界面相互作用来实现电致变色性能优化 ${ }^{[11]}$ 。 复合纳米材料通常可以兼顾两者甚至更多的优越性 能，同时达到弱化单一材料局限性的效果 ${ }^{[12-13]}$ 。

本工作采用水热法在 FTO 导电玻璃上制备了 垂直生长的 $\mathrm{NiO}$ 纳米片薄膜。用电沉积法在 $\mathrm{NiO}$ 薄 膜上复合 $\mathrm{PB}$, 制得 $\mathrm{NiO} / \mathrm{PB}$ 复合薄膜, 并对 $\mathrm{NiO}$ 薄 膜和 $\mathrm{NiO} / \mathrm{PB}$ 复合薄膜的电化学性能和电致变色性 能进行了测试和分析。

\section{1 实验方法}

\section{$1.1 \mathrm{NiO}$ 晶种层的制备}

将 FTO 导电玻璃依次放入玻璃清洗剂、去离子 水、丙醞、乙醇中各超声清洗 $20 \mathrm{~min}$, 烘干备用。 取 $0.6 \mathrm{~g}$ 四水乙酸镍溶于 $12 \mathrm{~mL}$ 体积比为 $1: 1$ 的无 水乙醇和正丁醇的混合溶液中, 搅拌至完全溶解。 向溶液中逐滴加入氨水直至溶液变成深绿色, 后将 此溶液旋涂在干净的 $\mathrm{FTO}$ 导电玻璃表面, $60^{\circ} \mathrm{C}$ 真空 干燥 $12 \mathrm{~h}$ 得到氢氧化镍晶种层。

\section{$1.2 \mathrm{NiO}$ 薄膜的制备}

取 $0.6 \mathrm{~g}$ 四水乙酸镍溶于 $56 \mathrm{~mL}$ 去离子水中搅 拌至固体完全溶解，后向澄清溶液中加入 $0.3 \mathrm{~g}$ 过硫 酸钾和 $3 \mathrm{~mL}$ 氨水, 搅拌 $40 \mathrm{~min}$, 转移到 $80 \mathrm{~mL}$ 水热 釜内胆中, 得到水热反应溶液。将负载有氢氧化镍 晶种层的 FTO 导电玻璃浸入盛有反应溶液的水热 釜内胆中, $180^{\circ} \mathrm{C}$ 保温反应 $2 \mathrm{~h}$ 。反应结束后自然冷却 到室温, 取出导电玻璃, 用水和乙醇依次清洗后自 然干燥制得 $\mathrm{NiO}$ 薄膜。

\section{$1.3 \mathrm{NiO} / \mathrm{PB}$ 复合电致变色薄膜的制备}

将 $0.67 \mathrm{~g}$ 六水三氯化铁、 $0.83 \mathrm{~g}$ 铁氰化钾以及 $0.53 \mathrm{~g}$ 无水氯化锂溶于 $250 \mathrm{~mL}$ 去离子水中, 磁力摚 拌至完全溶解, 制得 PB 电沉积反应溶液。电化学沉 积采用三电极体系, 以 $\mathrm{NiO}$ 薄膜作为工作电极, $\mathrm{Ag} / \mathrm{AgCl}$ 和铂片电极分别作为参比电极和对电极, 外加恒电流- $0.0005 \mathrm{~A}$, 沉积时间为 $150 \mathrm{~s}$ 。反应结束 后取出导电玻璃, 用水和乙醇依次清洗后自然干燥 制得 $\mathrm{NiO} / \mathrm{PB}$ 复合电致变色薄膜。

\section{4 电致变色器件的组装}

分别以 $\mathrm{NiO}$ 薄膜和 $\mathrm{NiO} / \mathrm{PB}$ 复合薄膜作为电致 变色电极, 以 FTO 导电玻璃作为对电极, 电极之间 用玻璃胶封装四边, 然后用一次性注射器将 $1 \mathrm{~mol} / \mathrm{L}$ $\mathrm{LiClO}_{4}-\mathrm{PC}$ 电解液注入到电极之间, 注入电解液的同 时将空间内的气泡排出。具体封装流程如图 1 所示。

\section{5 样品表征与性能测试}

采用日本 Rigaku 公司的 D/max-2550 型 X 射线 衍射仪分析产物物相，射线源为 $\mathrm{Cu}-\mathrm{K} \alpha$ (管压 $40 \mathrm{kV}$, 电流 $300 \mathrm{~mA}, \lambda=0.154056 \mathrm{~nm}$ ), 扫描速度为 $0.02 \%$, 扫描范围为 $5^{\circ} \sim 90^{\circ}$ 。采用日本 Hitachi 公司的 Model S-4800 场发射扫描电子显微镜观察电致变色薄膜的 微观形貌。采用上海辰华公司的 CHI 760D 电化学



图 1 电致变色器件组装示意图

Fig. 1 Schematic illustration for the electrochromic device preparation process 
工作站以及美国 Perkin Elmer 公司的 Lambda 950 紫外-可见分光光度计对电致变色薄膜和器件的电 化学性能以及电致变色性能进行测试。采用三电极 体系测试电化学性能, 以铂片为对电极, $\mathrm{Ag} / \mathrm{AgCl}$ 为 参比电极, 电致变色薄膜作为工作电极, $1 \mathrm{~mol} / \mathrm{L}$ 高 氯酸锂的碳酸丙烯酯溶液作为电解液。

\section{2 结果与讨论}

\section{$2.1 \mathrm{NiO}$ 以及 $\mathrm{NiO} / \mathrm{PB}$ 薄膜的物相分析}

图 2 为水热法制备 $\mathrm{NiO}$ 薄膜的 XRD 图谱。排 除 FTO 导电玻璃晶型的影响, 图 2(a)中的三个衍射 峰与立方相的 $\mathrm{NiO}$ (cubic, JCPDS 47-1049)的特征衍 射峰相对应, 且没有多余杂峰, 说明所制得的 $\mathrm{NiO}$ 薄膜为立方晶相。

从图 3 中可以看出 $\mathrm{Fe}$ 和 $\mathrm{Ni}$ 元素均匀分布在 $\mathrm{NiO} / \mathrm{PB}$ 复合电致变色薄膜表面。图 4 为 $\mathrm{NiO} / \mathrm{PB}$ 复 合电致变色薄膜表面 EDS 能谱分析, 结果显示复合 薄膜中存在 PB 薄膜的所有组成元素。结合 $\mathrm{Fe}$ 元素的 均匀分布, 说明 $\mathrm{PB}$ 已经成功结合到 $\mathrm{NiO}$ 薄膜表面。

\section{$2.2 \mathrm{NiO}$ 以及 $\mathrm{NiO} / \mathrm{PB}$ 薄膜的形貌分析}

图 5 为 $\mathrm{NiO}$ 薄膜和 $\mathrm{NiO} / \mathrm{PB}$ 复合薄膜的场发射 扫描电镜照片。从图 $5(\mathrm{a}, \mathrm{b})$ 可以看出, 水热法制备

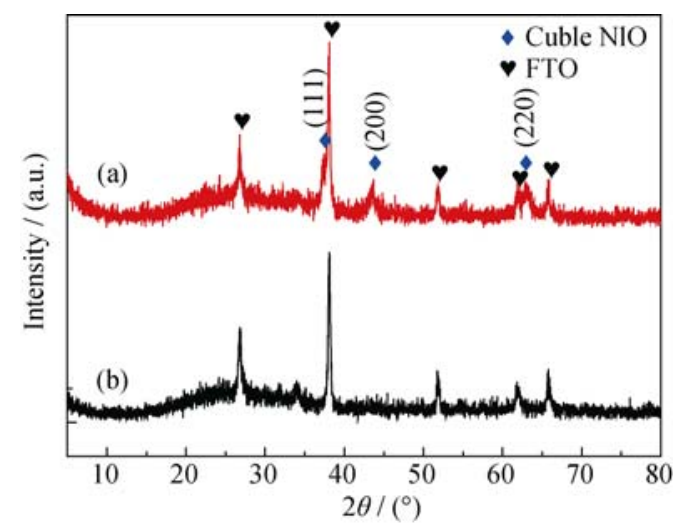

图 2 (a) $\mathrm{NiO}$ 薄膜和(b) FTO 玻璃的 XRD 图谱

Fig. 2 XRD patterns of (a) as-prepared NiO film and (b) FTOglass
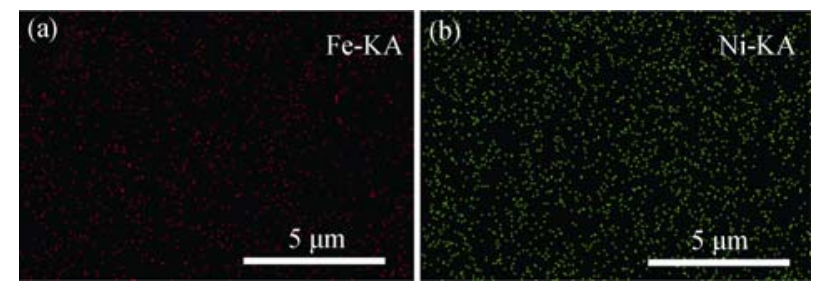

图 $3 \mathrm{NiO} / \mathrm{PB}$ 复合薄膜表面(a) $\mathrm{Fe}$ 和(b) $\mathrm{Ni}$ 元素分布图

Fig. 3 EDS mapping of $\mathrm{Fe}$ and Ni elements in the surface of $\mathrm{NiO} / \mathrm{PB}$ film

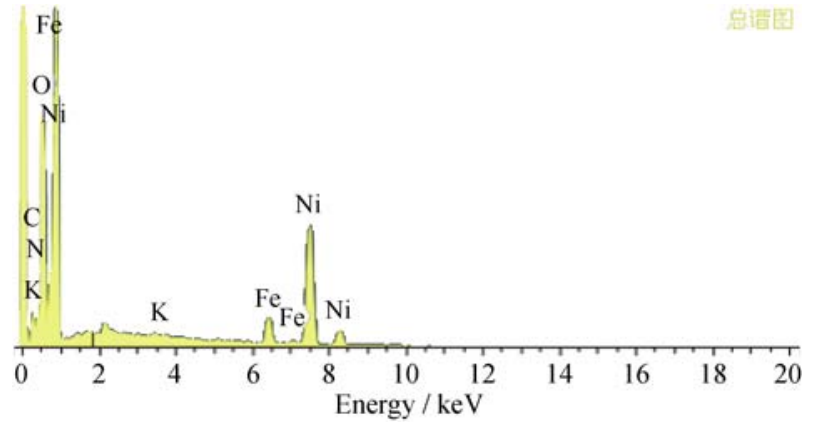

图 $4 \mathrm{NiO} / \mathrm{PB}$ 复合薄膜表面 $\mathrm{EDS}$ 能谱分析结果

Fig. 4 EDS analysis of the NiO/PB film



图 $5 \mathrm{NiO}$ 薄膜 $(\mathrm{a}, \mathrm{b})$ 和 $\mathrm{NiO} / \mathrm{PB}$ 薄膜 $(\mathrm{c}, \mathrm{d})$ 的 SEM 照片

Fig. 5 FESEM morphologies of $\mathrm{NiO}$ film $(\mathrm{a}, \mathrm{b})$ and $\mathrm{NiO} / \mathrm{PB}$ films (c, d)

的薄膜由交错生长的 $\mathrm{NiO}$ 纳米片组成。所得到的 $\mathrm{NiO}$ 薄膜具有多孔结构, 比表面积较大，可以明显 增大电解液和电致变色薄膜的接触面积, 加快电解 液与电致变色层之间的离子传输速率，明显提升电 致变色性能。图 $5(\mathrm{c}, \mathrm{d})$ 是电沉积得到的 $\mathrm{NiO} / \mathrm{PB}$ 复 合薄膜的场发射扫描电镜照片, 从图中可以看出, $\mathrm{NiO}$ 薄膜的孔洞结构都被完整地保留下来, 但是片 状结构的厚度增加, 说明经过电沉积, PB 已成功负 载到 $\mathrm{NiO}$ 薄膜上。对比图 5 (b)和(d)可以看出, $\mathrm{NiO} / \mathrm{PB}$ 复合薄膜的片状结构破损的情况明显轻于 $\mathrm{NiO}$ 薄膜, 这说明 $\mathrm{PB}$ 负载到 $\mathrm{NiO}$ 薄膜可以明显地 提高薄膜的机械稳定性, 保证多孔结构的稳定性, 有望获得更好的电致变色性能。

\section{$2.3 \mathrm{NiO}$ 薄膜以及 $\mathrm{NiO} / \mathrm{PB}$ 薄膜的电化学和电 致变色性能}

图 6 为 $\mathrm{NiO}$ 薄膜和 $\mathrm{NiO} / \mathrm{PB}$ 薄膜的循环伏安曲 线图, 从图中可以得出, $\mathrm{NiO} / \mathrm{PB}$ 薄膜的阳极和阴极 电流峰值相对于 $\mathrm{NiO}$ 薄膜都有很大地提高, 其中阳 极电流密度提高了 $24 \%$, 阴极峰值电流密度提高了 $38 \%$ 。 $\mathrm{NiO}$ 与 $\mathrm{PB}$ 复合后电位发生了变化, 说明 $\mathrm{NiO}$ 
与 $\mathrm{PB}$ 之间存在一定协同作用, 而不是简单的物理 结合, 这主要是由于薄膜电阻增大造成的 ${ }^{[8]}$ 。PB 负 载到 $\mathrm{NiO}$ 薄膜上, 不仅使纳米片的厚度增加, 而且 $\mathrm{PB}$ 与 $\mathrm{NiO}$ 材料之间产生了界面电阻。复合薄膜的 氧化还原过程需要在更高的电压驱动下实现, 但是 由于 PB 具有立方晶格结构, 搭建起来的三维网状 结构可用作离子和电子的传输介质和通道, 并且复 合薄膜呈现多孔结构, 具有较大的比表面积, 有利 于增大电解质与电极材料的接触面积, 缩短离子扩 散路径, 因此复合薄膜体现出更大的峰值电流密度。

为了测试 $\mathrm{NiO}$ 薄膜以及 $\mathrm{NiO} / \mathrm{PB}$ 薄膜的电致变 色性能, 将电致变色薄膜组装成电致变色器件进行 测试。器件的组装结构如下: glass/FTO/NiO/ $/ \mathrm{LiClO}_{4}$ $\mathrm{PC} / \mathrm{FTO} /$ glass(ECD-1), glass/FTO/NiO/PB/LiClO $4-\mathrm{PC} /$ $\mathrm{FTO} / \mathrm{glass}(\mathrm{ECD}-2)$ 。图 7 为电致变色器件在 $-3 \mathrm{~V}$ 裉色, $2.5 \mathrm{~V}$ 着色测试条件下的紫外-可见光透过率 曲线图, 从图中可以看出, 在可见光波长为 $700 \mathrm{~nm}$ 处, ECD-1 的光调制范围约为 33\%, 而 ECD-2 的光 调制范围可以达到 $46 \%$ 。在 $550 \mathrm{~nm}$ 波长处进行了 透过率对比, 结果显示 $\mathrm{NiO} / \mathrm{PB}$ 复合薄膜的光调制 范围比 $\mathrm{NiO}$ 薄膜提高了 $50 \%$ 左右, 这主要是因为 $\mathrm{PB}$ 作为阳极电致变色材料, 其本身具有光调制范围大的 优点, 复合薄膜可以同步发生变色过程, 因此体现出 比单层 $\mathrm{NiO}$ 薄膜更大的光调制范围。

图 8 是 ECD-1 和 ECD-2 在着色态 $(2.5 \mathrm{~V}, 60 \mathrm{~s})$ 和裉色态 $(-3 \mathrm{~V}, 60 \mathrm{~s})$ 的原位紫外-可见光透过率变 化曲线图, 测试选取的波长为 $700 \mathrm{~nm}$, 电致变色响 应时间以透过率为 $90 \%$ 的变化所需的时间为响应时 间。根据图 8 可以计算出 ECD-1 的着色时间和褪色 时间分别为 $15 \mathrm{~s}$ 和 $10 \mathrm{~s}, \mathrm{ECD}-2$ 的着色时间和褪色 时间分别为 $5 \mathrm{~s}$ 和 $6 \mathrm{~s}$ 。通过对比可以看出, $\mathrm{NiO} / \mathrm{PB}$ 薄膜的电致变色响应速度明显地提高, 这是因为

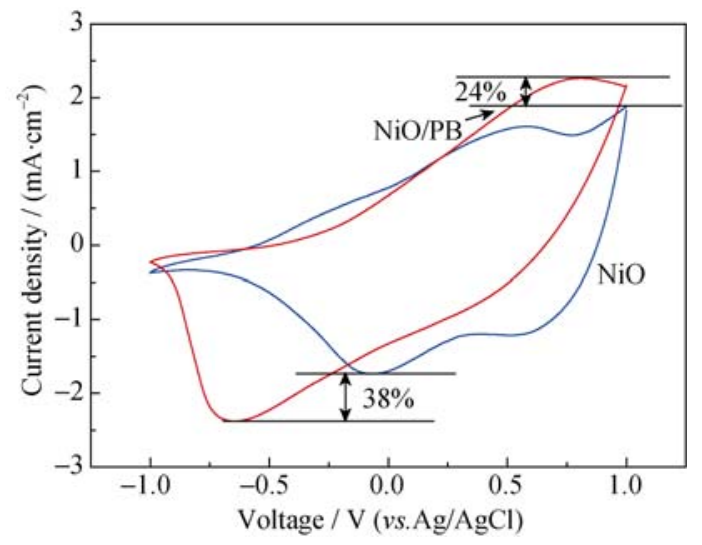

图 $6 \mathrm{NiO}$ 薄膜和 $\mathrm{NiO} / \mathrm{PB}$ 薄膜的循环伏安曲线图 Fig. 6 Cyclic voltammetry of $\mathrm{NiO}$ and $\mathrm{NiO} / \mathrm{PB}$ films
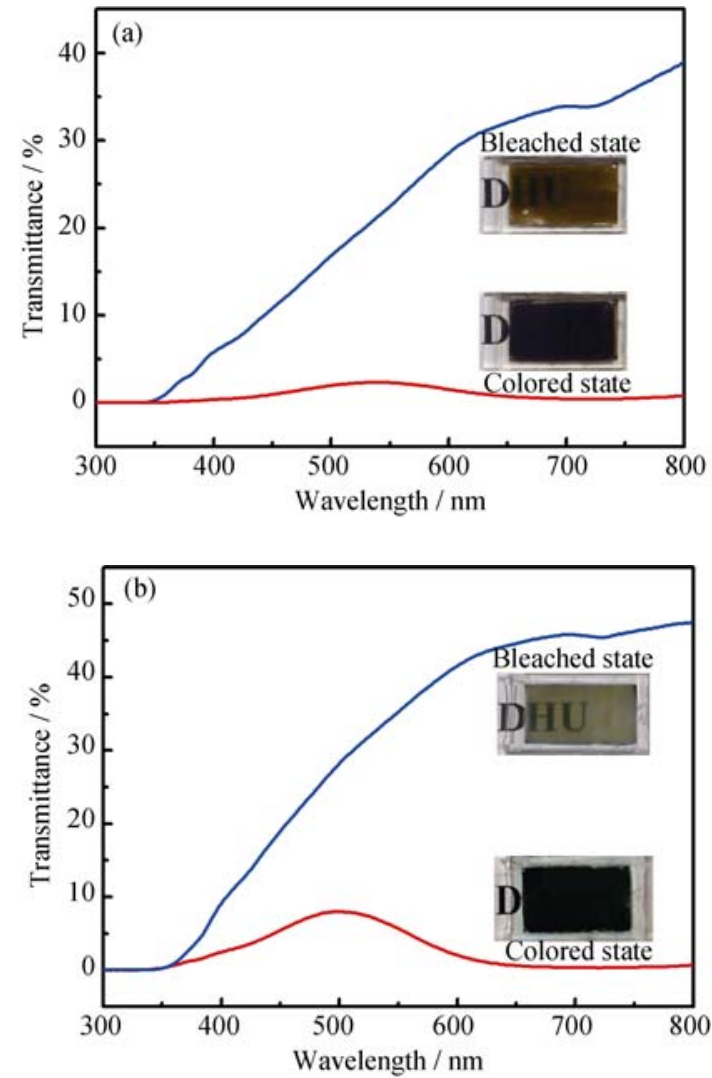

图 7 ECD-1 (a)和 ECD-2(b)在着色态和褪色态的紫外-可见 光透过率曲线

Fig. 7 UV-Vis transmittance spectra of as-prepared ECD-1 (a) and ECD-2 (b)

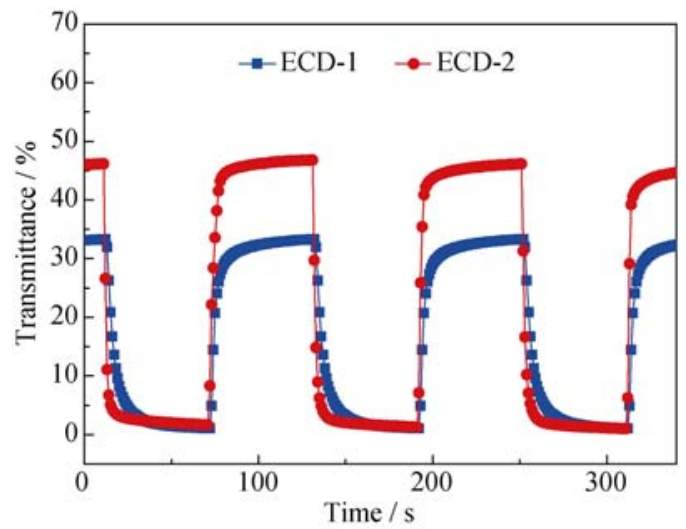

图 8 ECD-1 和 ECD-2 在着色态和褪色态的原位光透过率变 化曲线图

Fig. 8 Switching time characteristics between the colored and bleached states for ECD-1 and ECD-2 measured at $700 \mathrm{~nm}$ with potential of $-3 \mathrm{~V}$ and $2.5 \mathrm{~V}$

$\mathrm{PB}$ 的内部晶格结构为离子和电子的传输提供了通 道; 其次, $\mathrm{Fe}^{2+}$ 与 $\mathrm{Fe}^{3+}$ 作为立方晶格的顶点, 暴露在 晶格外部, 利于电荷的转移, 加快电荷转移和离子 扩散速度。

图 9 为 ECD-1 和 ECD-2 光密度变化值随着注 

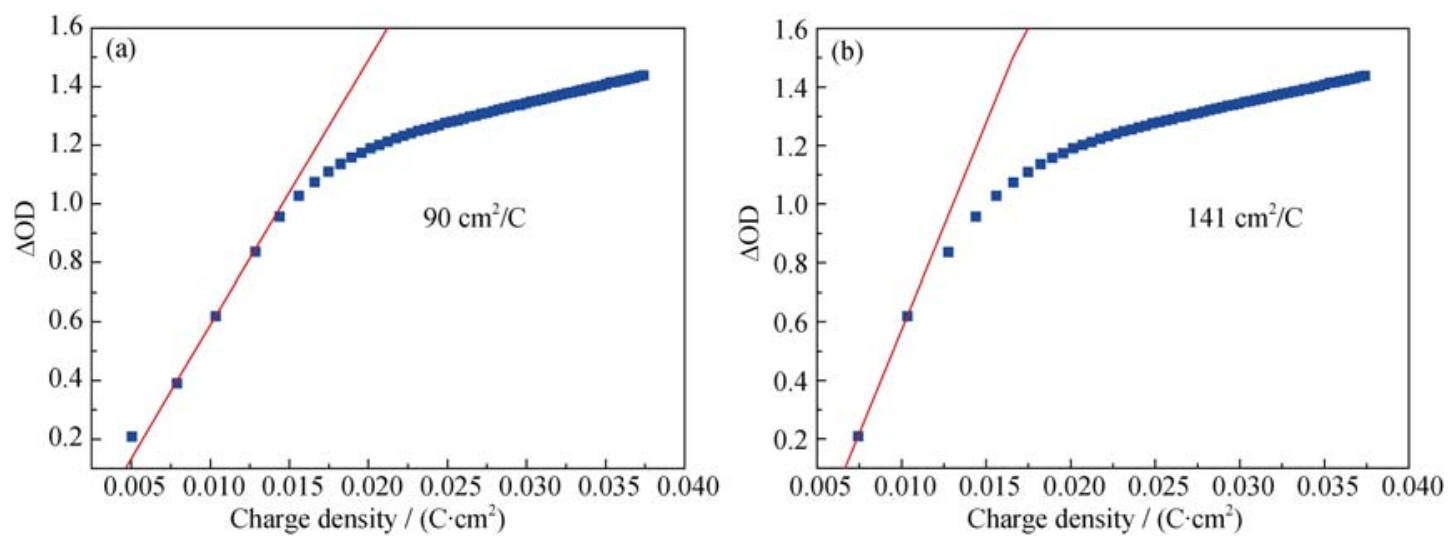

图 9 (a) ECD-1 和(b) ECD-2 电致变色器件的光密度变化值随注入电荷密度的变化曲线图

Fig. 9 OD variation with respect to the charge density for (a) ECD-1 and (b) ECD-2 measured at $700 \mathrm{~nm}$ with a potential of $2.5 \mathrm{~V}$

入电荷密度的变化曲线图, 按照着色效率计算公式, 可以计算出 ECD-1 和 ECD-2 在 $2.5 \mathrm{~V}$ 电压下的着色 效率分别为 90 和 $141 \mathrm{~cm}^{2} / \mathrm{C}$ 。交错且直立生长的片 状结构为离子传输过程提供了较大的反应活性面积, 加快了离子的嵌入迁出速度, 因此 ECD-1 可以得到 较高的着色效率。ECD-2 可以实现更高的着色效率, 这主要是因为 $\mathrm{NiO}$ 和 $\mathrm{PB}$ 同为阳极电致变色材料, 可以实现同步着色过程。并且 $\mathrm{PB}$ 具有较高的离子 传输能力, 即便是在较低的外加电压作用下, 也可 以实现较快的着色响应，从而显著提高复合薄膜的 着色效率。

采用计时电流法测试 ECD-1 和 ECD-2 的阳极 和阴极峰值电流密度与循环次数之间的关系, 对器 件外加方波电压为 $2.5 \mathrm{~V}$ (着色态, $10 \mathrm{~s}$ ) 和 $-3.0 \mathrm{~V}$ (褪色 态，10 s)测试其阴极和阳极的峰值电流密度变化, 测试结果如图 10 所示。从图 10 可以看出, 在经过 1000 次着色褪色循环之后, ECD-1 和 ECD-2 的阳极 和阴极峰值电流密度均有不同程度的衰减。但是在

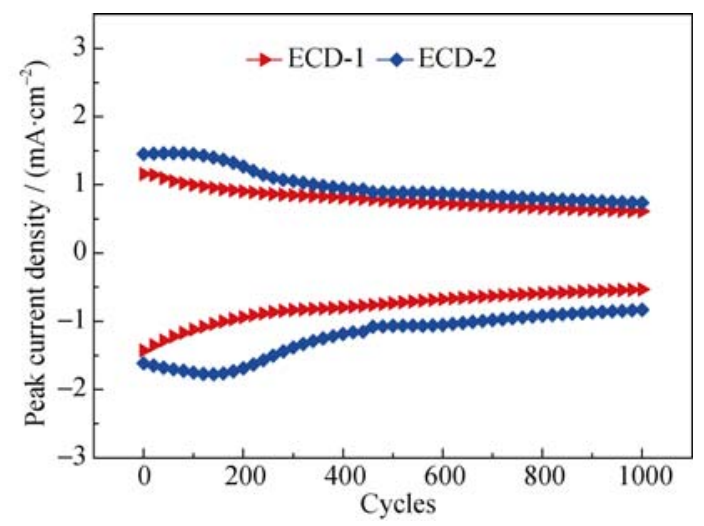

图 10 ECD-1 和 ECD-2 计时电流曲线的峰值电流密度随循环 次数的变化关系

Fig. 10 Peak current evolution of the ECD-1 and ECD-2 during the step chronoamperometric cycles
1000 次变色循环过程中, ECD-2 始终保持较高的峰 值电流密度。ECD-2 经过 1000 次循环之后, 电致变 色薄膜并未发生明显的脱落或是着色不均匀的现象, 但是电解液消耗严重, 器件内气泡较多。实验结果 说明, 较厚的薄膜结构不利于 $\mathrm{Li}^{+}$嵌入和迁出, 并且 由于 $\mathrm{NiO}$ 片状结构的厚度以及晶格结构的限制, $\mathrm{Li}^{+}$ 的嵌入和迁出过程都不彻底, 电致变色薄膜内部会 残余未及时迁出的 $\mathrm{Li}^{+}$, 从而导致电解液的严重消 耗, 这也大大降低了器件的循环使用寿命。

\section{3 结论}

通用水热法和电沉积法制备了 $\mathrm{NiO} / \mathrm{PB}$ 复合薄 膜。相比于 $\mathrm{NiO}$ 薄膜, $\mathrm{NiO} / \mathrm{PB}$ 复合薄膜具有更高的 峰值电流密度、更大的光调制范围(700 nm, 46\%)、 更高的着色效率 $\left(141 \mathrm{~cm}^{2} / \mathrm{C}\right)$ 以及更快的变色响应速 度 $\left(t_{\mathrm{b}}=6 \mathrm{~s}, t_{\mathrm{c}}=5 \mathrm{~s}\right)$ 。由此可见, $\mathrm{NiO} / \mathrm{PB}$ 复合薄膜是一种 新型的阳极电致变色薄膜，具有良好的应用前景。

\section{参考文献:}

[1] KNODALKAR V V, PATIL P B, MANE R M, et al. Electrochromic performance of nickel oxide thin film: synthesis via electrodeposition technique. Macromolecular Symposia., 2016, 361(1): $47-50$.

[2] DONG D M, WANG W W, DONG G B, et al. Electrochromic properties and performance of $\mathrm{NiO}_{x}$ films and their corresponding all-thin-film flexible devices prepared by reactive DC magnetron sputtering. Applied Surface Science, 2016, 383: 49-56.

[3] NAKAOKA K, UEYAMA J, OGURA K. Semiconductor and electrochromic properties of electrochemically deposited nickel oxide films. Journal of Electroanalytical Chemistry, 2004, 571(1): 93-99.

[4] LEE S, PARK S, KIM C W, et al. Defect-related photolumines- 
cence properties of as-synthesized and annealed $\mathrm{NiO}$ nanostructures via hydrothermal method. Thin Solid Films, 2016, 598: 33- 38.

[5] MA D Y, SHI G Y, WANG H Z, et al. Hierarchical NiO microflake films with high coloration efficiency, cyclic stability and low power consumption for applications in a complementary electrochromic device. Nanoscale, 2013, 5(11): 4808-4815.

[6] WANG RONG, WU XIA-QIN, HUANG CHUN-QIN, et al. Study on the electrochemical features of Prussian blue film and copper ferricyanide composite film. Journal of Shanghai Teachers University (Natural Science), 2001, 30(1): 57-60.

[7] QIU SI-CHOU, LIU HUI, HUANG HAN-YAO, et al. The electrochromic device of Prussian blue film. Functional Materials, 1992, 23(1): 50-53.

[8] LI T L, ZHAO C C, MA D Y, et al. Electrodeposition of Prussian blue films on $\mathrm{Ni}_{3} \mathrm{Si}_{2} \mathrm{O}_{5}(\mathrm{OH})_{4}$ hollow nanospheres and their enhanced electrochromic properties. RSC Advances, 2016, 6(46): 39833-39838.

[9] HONG S F, CHEN L C. Nano-Prussian blue analogue/PEDOT:
PSS composites for electrochromic windows. Solar Energy Materials and Solar Cells, 2012, 104: 64-74.

[10] ASSIS L M N, ANDRADE J R, SANTOS L H E, et al. Spectroscopic and microscopic study of Prussian blue film for electrochromic device application. Electrochimica Acta, 2015, 175: 176-183.

[11] YAO D D, RANI R A, O'MULLANE A P, et al. Enhanced coloration efficiency for electrochromic devices based on anodized $\mathrm{Nb}_{2} \mathrm{O}_{5} /$ electrodeposited $\mathrm{MoO}_{3}$ binary systems. The Journal of Physical Chemistry C, 2014, 118(20): 10867-10873.

[12] DELONGCHAMP D M, HAMMOND P T. Multiple-color electrochromism from layer-by-layer-assembled polyaniline/Prussian blue nanocomposite thin films. Chemistry of Materials, 2004, 16(23): 4799-4805.

[13] KIM T H, JEON H J, LEE J W, et al. Enhanced electrochromic properties of hybrid $\mathrm{P} 3 \mathrm{HT} / \mathrm{WO}_{3}$ composites with multiple colorations. Electrochemistry Communications, 2015, 57: 65-69. 